\title{
All optically tunable wavelength-selective reflector consisting of coupled polymeric microring resonators
}

\author{
Jacob Scheuer ${ }^{\mathrm{a})}$ \\ Center for the Physics of Information, Applied Physics, California Institute of Technology, MC 128-95, \\ Pasadena, California 91125 \\ George T. Paloczi and Amnon Yariv \\ Department of Applied Physics, California Institute of Technology, Pasadena, California 91125 \\ (Received 17 January 2005; accepted 25 October 2005; published online 13 December 2005) \\ We present an all optically tunable wavelength-selective reflector for planar lightwave technology \\ based on coupled microring resonators. By employing the Vernier effect, we demonstrate \\ narrow-band reflection and strong side-lobe suppression in an optical polymer device fabricated by \\ soft lithography. Wide and simple tuning of the reflection peak using an external control beam is \\ demonstrated. (C) 2005 American Institute of Physics. [DOI: 10.1063/1.2149158]
}

Semiconductor lasers that exhibit stable wavelength and narrow bandwidth are key elements for modern optical communication systems. ${ }^{1}$ For wavelength division multiplexing (WDM) applications, it is also desired that the laser wavelength be reconfigurable or trimable. In most lasers for WDM, the demand for stable lasing wavelength is fulfilled by employing either Bragg reflectors ${ }^{2}$ or external cavities. ${ }^{3,4}$ However, employment of distributed Bragg reflectors (DBRs) requires additional high-resolution lithography steps over relatively large areas in order to achieve high reflection efficiency and low scattering losses. ${ }^{5}$ This is especially true for applications requiring aperiodic or apodized reflection gratings. In addition, tuning the reflection wavelength of a DBR over wide range without deteriorating the reflection characteristics is not simple and requires careful design and implementation. The integration of reflectors into planar optical circuits is highly advantageous, enabling compact re- flectors that can be realized using a wide variety of materials, and integration with additional on-chip photonic devices, such as modulators, couplers, sources, etc.

In this letter, we present and demonstrate an alternative configuration for an in-plane, tunable, and wavelengthselective reflector which is based on coupled microring resonators. Although microring resonators had been extensively studied for a variety of applications, ${ }^{6-12}$ the use of ring resonators as reflectors received less attention ${ }^{13,14}$ and tunable reflection using microring resonators have not been demonstrated.

Figure 1 shows an optical image of the device. Light is launched into the input waveguide and is equally divided between the two arms of the $Y$ junction. The signal in each arm is filtered twice by passing through the cascaded microring add/drop filters. The reflection function of the composite filter is given by:

$$
D(\omega)=\frac{k_{1} k_{2}\left(1-L_{1}\right)^{1 / 4}\left(1-L_{2}\right)^{1 / 4} e^{i \pi R_{1} n_{\mathrm{eff}} \omega} e^{i \pi R_{2} n_{\mathrm{eff}} \omega}}{\left[1-\left(1-k_{1}\right) \sqrt{1-L_{1}} e^{i 2 \pi R_{1} n_{\mathrm{eff}} \omega}\right] \cdot\left[1-\left(1-k_{2}\right) \sqrt{1-L_{2}} e^{i 2 \pi R_{2} n_{\mathrm{eff}} \omega}\right]},
$$

where $k_{i}, L_{i}$, and $R_{i}$ are, respectively, the power coupling, loss per revolution, and the radius of the $i \operatorname{ring}(i=1,2)$. Note that the transfer function (1) is independent of the order of the two rings and, therefore, the light in both arms experiences identical filtering. Finally, the two parts of the filtered signal are combined by the $Y$ splitter to generate the reflected signal. The remaining signal, which is not within the bandwidth of the filter, continues through Ports 2 and 3 (see Fig. 1) and can be used as a monitor for tuning the resonance wavelengths of the cavities.

The architecture of the device shown in Fig. 1 exhibits several inherent advantages. The reflected wavelengths must be resonant with all the microring cavities composing the filter, thus allowing for the reflection of a small set of wave-

\footnotetext{
${ }^{\text {a)} E l e c t r o n i c ~ m a i l: ~ k o b y @ c a l t e c h . e d u ~}$
}

lengths (or even a single wavelength) by using microrings with different radii (the Vernier effect). In addition, the bidirectional propagation architecture of the device ensures that the two parts of the signal traverse identical paths and, thus, no postfabrication balancing of the device is required.

Figure 2 shows the calculated spectral responses of the three ports for a device consisting of microrings with radii $R_{1}=125 \mu \mathrm{m}, R_{2}=100 \mu \mathrm{m}, k_{1}=k_{2}=0.3, L_{1}=0.3, L_{2}=0.25$ (corresponding to loss of $20 \mathrm{~dB} / \mathrm{cm}$ ) and group index of $n_{g}$ $=1.655$. The corresponding free spectral ranges $(\mathrm{FSR})$ of the resonators are $\mathrm{FSR}_{1}=230.8 \mathrm{GHz}$ and $\mathrm{FSR}_{2}=288.5 \mathrm{GHz}$. Because of the $4: 3$ ratio between the resonators radii, every fourth resonance of Ring 2 aligns with a resonance of Ring 1, generating an effective (collective) FSR of $1154 \mathrm{GHz}$. This can be clearly seen in Figs. 2(a) and 2(b) showing the reflected (Port 1) and transmitted (Port 2) spectra. 


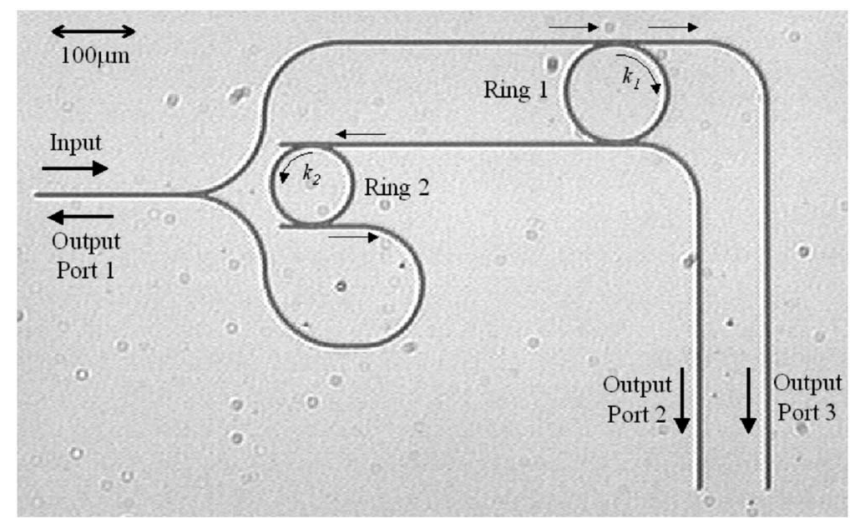

FIG. 1. An optical image of the proposed wavelength-selective reflector.

To demonstrate the concept of and the ability to tune the reflected wavelength, the devices are fabricated using the soft-lithography replica-molding method ${ }^{15,16}$ of a polymer doped with electro-optic chromophore CLD-1. ${ }^{17,18}$ The replica-molding method is a powerful technique since it does not affect the dopant molecules in the polymers, allowing for a wide variety of dopants to be introduced into the polymer matrix. The polymer solution used to mold the device consists of $5.5 \mathrm{wt} \%$ of CLD-1/amorphous polycarbonate (APC) in trichloroethylene/dibromomethane. The CLD-1:APC ratio is $1: 4$ by weight and the solvent consists of trichloroethylene and dibromomethane in a $50 \%$ ratio by volume. The radii of the microrings are $R_{1}=125 \mu \mathrm{m}, R_{2}=100 \mu \mathrm{m}$, the waveguide dimensions are approximately $1.6 \mu \mathrm{m} \times 2 \mu \mathrm{m}$ with a refractive index of $\sim 1.6 @ 1550 \mathrm{~nm}$. The lower and upper claddings are, respectively, $5 \mu \mathrm{m}$ thick thermally grown silicon oxide with refractive index of $\sim 1.445 @ 1550 \mathrm{~nm}$ and air. The separation between the waveguides and the microrings
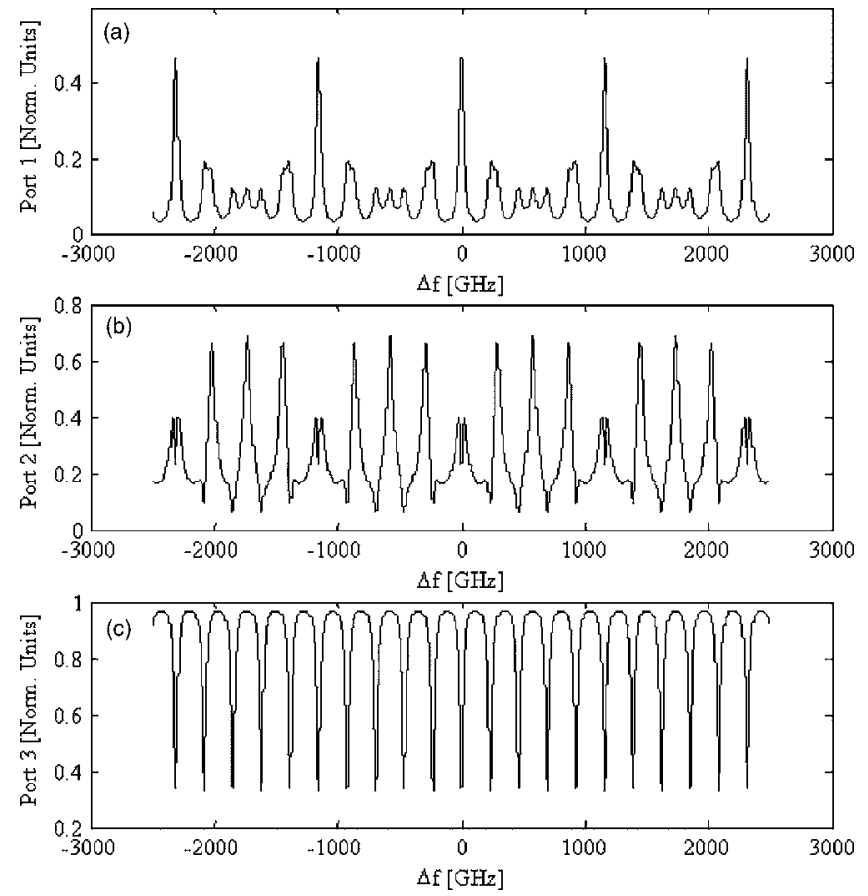

FIG. 2. Theoretically calculated spectra of the device: (a) Reflected signal, (b) Transmitted signal at Port 2, and (c) Transmitted signal at Port 3.

is approximately $400 \mathrm{~nm}$ with expected power coupling ratio of approximately 0.2 . The waveguide cross section supports a well-confined first mode and weakly confined second- and third-order modes which are expected to be quite lossy in the microring due to bending losses. The measured FSR of Ring $1(\sim 1.8 \mathrm{~nm})$ agrees well with the group index of this waveguide structure $\left(n_{\mathrm{g}} \sim 1.655\right)$, which was calculated using finite-difference mode solver software.
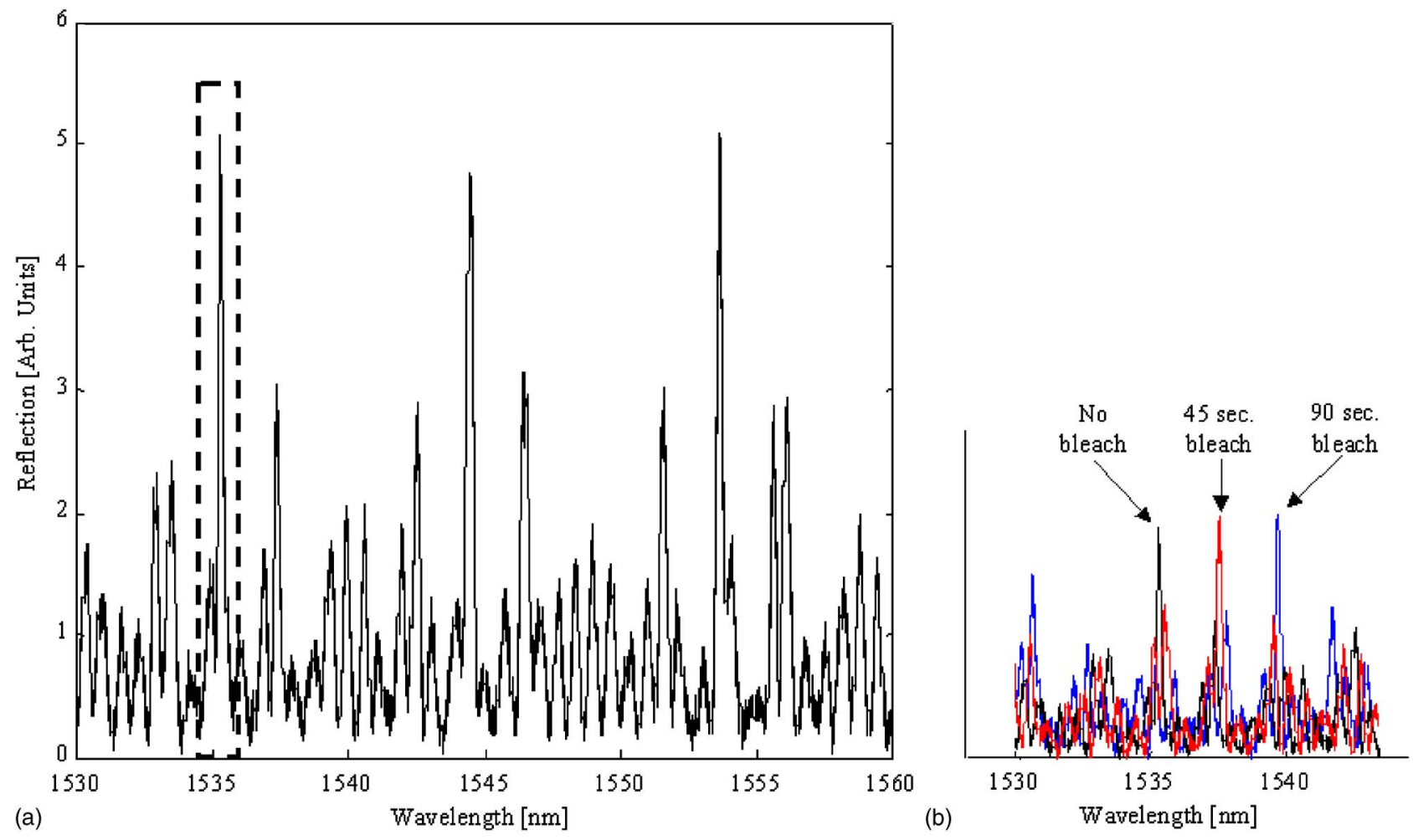

FIG. 3. (Color online) (a) Measured reflected spectrum at Port 1 and (b) tuning the reflection peak over more than 5 nm: No bleach (black); $45 \mathrm{~s}$ bleach (red), and $90 \mathrm{~s}$ bleach (blue).

Downloaded 13 Jan 2006 to 131.215.240.9. Redistribution subject to AIP license or copyright, see http://apl.aip.org/apl/copyright.jsp 
Transverse electric polarized light from a tunable laser is coupled to the device through a tapered fiber. The input signal passes through an on-chip implemented $3 \mathrm{~dB}$ coupler (before passing through the $Y$ splitter) in order to separate between the injected and the reflected signals. All signals (transmitted and reflected) are collected and focused on a photodetector using an objective lens. The input wavelength is scanned from $1530 \mathrm{~nm}$ to $1560 \mathrm{~nm}$ and the output signals are recorded as shown in Fig. 3.

Figure 3(a) depicts the reflected spectrum measured at the output of Port 1 . The good agreement between the measured reflection and the theory [see Fig. 2(a)] is evident: On the two sides of each mutual resonance, frequencies of both rings there are lower reflection peaks. The region between these triplets is occupied by small oscillations. The measured intensity ratio between the amplitudes of the main reflection peaks and those of the satellite lobes [Fig. 3(a)] is $\sim 1.67$ which is in good agreement with the predicted ratio of 1.75 .

To tune the reflected spectrum, we illuminate the rings with a focused broadband visible light beam. The illumination photobleaches the CLD-1 chromophores doping the polymer waveguides and changes their index of refraction, thus shifting the resonance wavelengths of the microrings. The rate of the shift can be controlled by varying the intensity of the illumination and the exposure area. In our experiment, the control beam spot covers completely the tuned ring in order to achieve uniform change in the refractive index along the whole ring. Figure 3(b) shows the tuning of one of the main reflection peaks (marked by the dashed rectangular) by illuminating ring 1 (see Fig. 1). After photobleaching Ring 1 for approximately $45 \mathrm{~s}$, the resonance wavelength of the microring shifts by $\sim 0.46 \mathrm{~nm}$ (the difference between the FSRs of the rings), resulting in an overall shift of $2.3 \mathrm{~nm}$ in the reflection peak from $\lambda=1535 \mathrm{~nm}$ to $\lambda=1537.3 \mathrm{~nm}$ (red spectrum). An additional $45 \mathrm{~s}$ of bleaching Ring 1 shifts the reflection peak by additional $2.3 \mathrm{~nm}$ to $\lambda=1539.6 \mathrm{~nm}$ (blue spectrum). The higher reflection peak at $1532 \mathrm{~nm}$ of the blue spectrum is the right satellite lobe of another mutual resonance of the two microrings.

As an alternative to an integrated Bragg grating reflection filter, the microring-based reflector presents several advantages and increased flexibility. Most notably, the microring-based reflector is more compact than typical integrated waveguide gratings, allowing for simpler and more controlled fabrication, as well as for the possibility of incorporation with additional photonic devices. The employment of the Vernier effect provides several advantages which enhances the performance of the reflector. In addition to the extended FSR, the Vernier effect extends the possible tuning range and speed because reaching every possible wavelength requires only the ability to tune over the rings' FSRs.

Although here we demonstrated optically tunable reflector, the same device, employing a similar material system, can be tuned electro-optically by applying electric voltage across the rings after poling the chromophores in order to align them. The thermo-optic effect can also be used for a similar purpose although the tuning speed will be slower in this case.

To summarize, we present a simple wavelength-selective optically tunable reflector based on coupled microring resonators. The reflector can be used to attain narrow singlewavelength reflection or higher-order response which is suitable for use as tunable laser endmirrors. A wide tuning range of the reflection peak is possible by employing the Vernier effect. The fabrication of the reflector is straightforward and does not require high-resolution lithography or highly accurate etching steps. The use of a chromophore-doped polymer for the waveguides allows for simple and wide-range tuning of the microrings' resonance wavelengths and, correspondingly, the reflected wavelength either by photobleaching the chromophore or by the electro-optic effect.

The authors thank Y. Huang for fruitful discussions. The research was supported by the National Science Foundation, the Defense Advanced Research Projects Agency, and Hughes Research Laboratories.

${ }^{1}$ G. P. Agrawal, Fiber-Optic Communication Systems (Wiley, New York, 1977).

${ }^{2}$ A. Yariv, Optical Electronics in Modern Communications (Wiley, New York, 1997).

${ }^{3}$ T. Schrans, S. Sanders, and A. Yariv, IEEE Photonics Technol. Lett. 4, 323 (1992).

${ }^{4}$ J.-I. Hashimoto, T. Takagi, T. Kato, G. Sasaki, M. Shigehara, K. Murashima, M. Shiozaki, and T. Iwashima, J. Lightwave Technol. 21, 2002 (2003).

${ }^{5}$ M. H. Lim, T. E. Murphy, J. Ferrera, J. N. Damask, and H. I. Smith, J. Vac. Sci. Technol. B 17, 3208 (1999).

${ }^{6}$ R. Orta, P. Savi, R. Tascone, and D. Trinchero, IEEE Photonics Technol. Lett. 7, 1447 (1995).

${ }^{7}$ B. E. Little, S. T. Chu, H. A. Haus, J. Foresi, and J.-P. Laine, J. Lightwave Technol. 15, 998 (1997).

${ }^{8}$ P. Rabiei, W. H. Steier, C. Zhang, and L. R. Dalton, J. Lightwave Technol. 20, 1968 (2002).

${ }^{9}$ T. A. Ibrahim, W. Cao, Y. Kim, J. La, J. Goldhar, P.-T. Ho, and C. H. Lee, IEEE Photonics Technol. Lett. 15, 36 (2003).

${ }^{10}$ B. Liu, A. Shakouri, and J. E. Bowers, Appl. Phys. Lett. 79, 3561 (2001).

${ }^{11}$ Z. Bian, B. Liu, and A. Shakouri, IEEE J. Quantum Electron. 39, 859 (2003).

${ }^{12}$ J. K. S. Poon, J. Scheuer, and A. Yariv, IEEE Photonics Technol. Lett. 16, 1331 (2004).

${ }^{13}$ P. Rabiei and W. H. Steier, IEEE Photonics Technol. Lett. 15, 1255 (2003).

${ }^{14}$ G. T. Paloczi, J. Scheuer, and A. Yariv, IEEE Photonics Technol. Lett. 17, 390 (2005).

${ }^{15}$ Y. Huang, G. T. Paloczi, J. Scheuer, and A. Yariv, Opt. Express 11, 2452 (2003).

${ }^{16}$ G. T. Paloczi, Y. Huang, J. Scheuer, and A. Yariv, J. Vac. Sci. Technol. B 22, 1764 (2004).

${ }^{17}$ C. Zhang, L. R. Dalton, M.-C. Oh, H. Zahng, and W. H. Steir, Chem. Mater. 13, 3043 (2001).

${ }^{18}$ M. B. J. Diemeer, F. M. M. Suyten, E. S. Trommel, A. McDonald, J. M. Copeland, L. W. Jenneskens, and W. H. G. Horsthuis, Electron. Lett. 26, 379 (1990). 